\title{
Minor challenges: Modified diverticulectomy and myotomy for recurrent Zenker diverticulum
}

\author{
Peter C. Minneci, MD, and Douglas J. Mathisen, MD, Boston, Mass
}

$\mathrm{P}$ haryngoesophageal diverticulum or Zenker diverticulum is a relatively uncommon surgical problem. The cause of this entity has been debated but still remains inconclusive. The symptoms of dysphagia, regurgitation of undigested food, aspiration, noisy deglutition, halitosis, and voice changes usually prompt surgical correction. ${ }^{1}$ A variety of techniques have been proposed for its correction, including diverticulopexy, diverticulectomy, cricopharyngeal myotomy, and endoscopic stapling or lasering. All of these techniques have proponents and detractors. ${ }^{1-5}$

The most common technique to treat Zenker diverticulum has been combined diverticulectomy and cricopharyngeal myotomy. This approach has achieved excellent results, with an overall success rate of $95 \%$, a recurrence rate of $3.6 \%$, and a low mortality rate of $1.2 \% .^{5}$ This has been our standard approach, with equally good results. We have usually performed the diverticulectomy open over a $40 \mathrm{~F}$ to $50 \mathrm{~F}$ bougie. The resulting defect is closed with inverting 4-0 silk sutures. The adjacent esophageal muscle is closed over the repair for the second layer of closure. Subsequently, a myotomy is then performed at a remote site on the esophagus.

During a recent operation for a failed repair performed elsewhere, there was little available esophageal muscle for the second layer of closure after resecting the diverticulum and completing the myotomy. We chose to use the omohyoid muscle to buttress the diverticulectomy site and to separate the edges of the myotomy, with excellent results.

Failed operations for Zenker diverticulum pose challenging problems. Tissue planes are difficult to identify because of the previous diverticulectomy and myotomy. Knowing where the previous myotomy was performed can be difficult to determine and subject to inadvertent opening of the esophagus. By using the diverticulum as a guide to establish the correct plane for myotomy, one can easily perform this important part of the operation. Adequate tissues to cover the diverticulectomy site are often lacking.

The use of the nearby omohyoid muscle provides a secure closure, with little if any cosmetic or functional loss. We have used

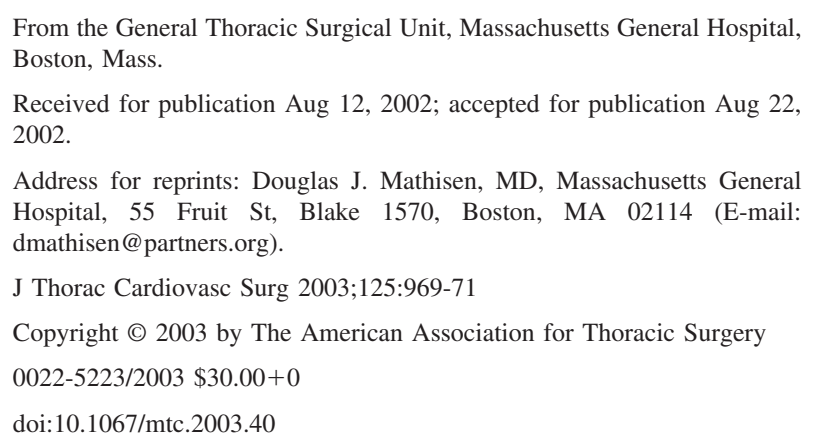

this approach in 3 reoperations and 2 primary repairs with good results.

\section{Methods and Technique}

We use a horizontal low-collar incision at the base of the neck rather than an incision along the sternocleidomastoid muscle. We believe this approach results in a superior cosmetic result with equal operative exposure. The dissection is carried down anterior to the carotid sheath to the prevertebral fascia. The omohyoid muscle is identified, retracted, and preserved for later use. The esophagus is identified, and care is taken not to retract in the tracheoesophageal groove to avoid injury to the recurrent laryngeal nerve. The diverticulum is identified and dissected circumferentially to the base of the neck of the diverticulum, freeing it from the surrounding tissues. The cricopharyngeus muscle can be identified as a tight muscular band at the base of the diverticulum (Figure 1, A). A myotomy is performed extending $3 \mathrm{~cm}$ below the diverticulum and proximally until all the cricopharyngeus muscle has been divided (Figure 1,B). The muscle is then separated from the mucosa anteriorly and posteriorly to allow separation of the muscle edges and to prevent recurrent narrowing by the cricopharyngeus. A $40 \mathrm{~F}$ to $50 \mathrm{~F}$ bougie is placed into the esophagus, and the neck of the sac is identified. The superior and inferior aspect of the diverticulum is marked with stay sutures, and the diverticulum is then transected at its neck. The bougie is then removed. The esophageal mucosa is then closed with inverted interrupted 4-0 silk sutures, with dunking of the last suture to create a linear closure (Figure 1,C). The integrity of the repair can be checked by pulling the nasogastric tube back and instilling air or methylene blue into the esophagus to check for leaks. The omohyoid muscle is then dissected free and mobilized into a superiorly or inferiorly based muscle flap. If the muscle is detached from the hyoid bone, sufficient length must be mobilized to avoid any compression of the internal jugular vein. The omohyoid muscle is then placed over the esophageal suture line and secured with multiple interrupted 4-0 silk sutures (Figure 1, D). The omohyoid muscle serves as a second layer and separates the cut edges of the cricopharyngeus muscle to prevent restenosis. The wound is irrigated, and hemostasis is achieved. A single drain is left in place, and the wound is closed in layers. A postoperative barium swallow is performed on day 3 .

\section{Results}

This approach has been used in 3 patients who had reoperations for persistent symptomatic Zenker diverticulum. There were no postoperative leaks or other complications. All patients were discharged by postoperative day 5 . All patients remain asymptomatic, with follow-up between 15 months and 6 years. 

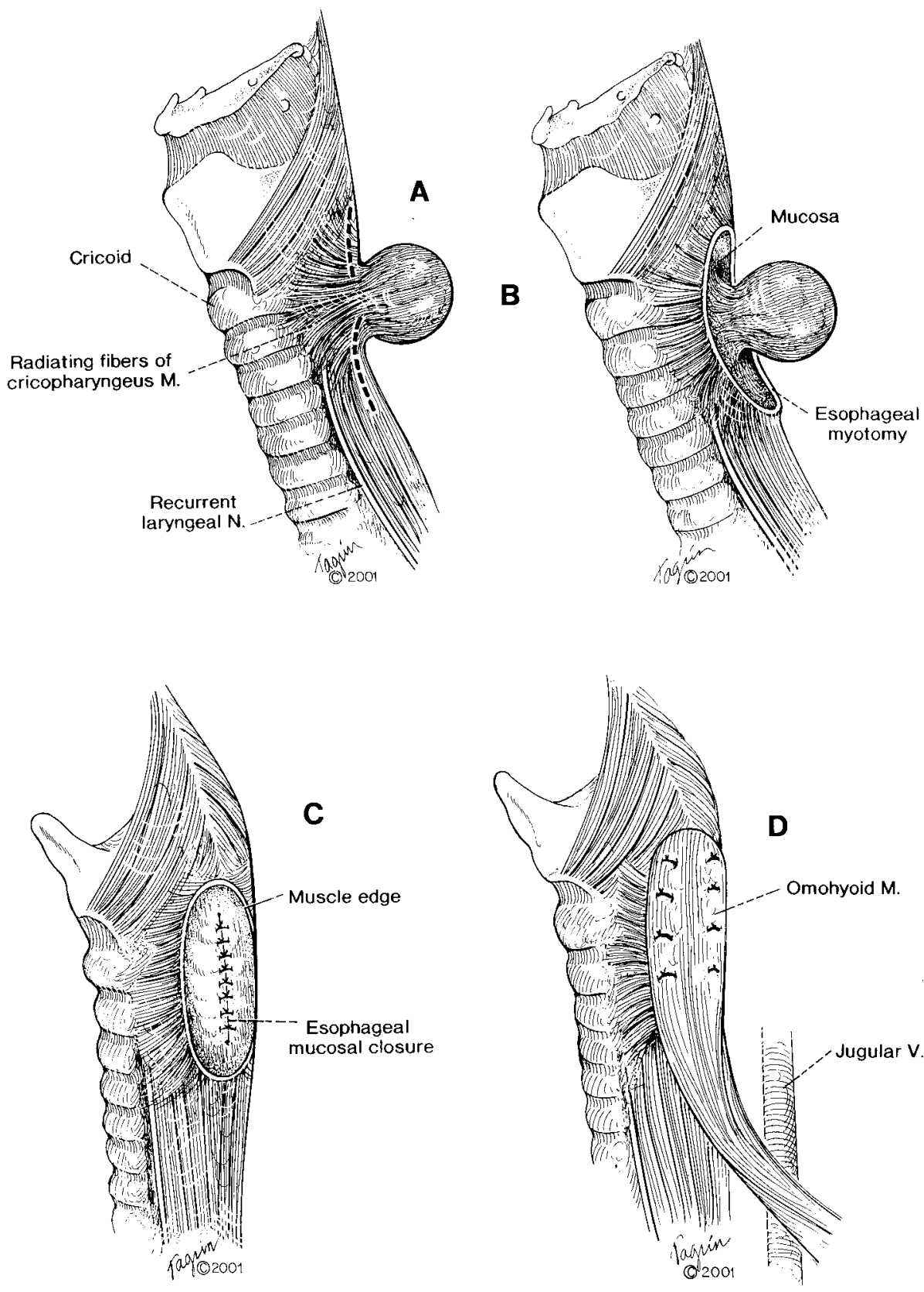

Figure 1. A, The diverticulum and cricopharyngeus muscle are identified. The dotted line represents the proposed myotomy. $M$, Muscle; $N$, nerve. B, The myotomy has been carried out, completely dividing the cricopharyngeus muscle. C, The diverticulum has been excised over a 40F to 50F Maloney bougie. The defect is closed with interrupted 4-0 silk sutures placed with knots on the inside to encourage an inverting closure. D, The omohyoid muscle is dissected to create an inferiorly based flap. It is divided near the hyoid bone. It is then carefully sutured to the edges of the divided cricopharyngeus muscle. This serves as a second-layer closure and to separate the edges of the muscle. $M$, Muscle; $V$, vein.

\section{Comment}

Successful correction of a Zenker diverticulum has been achieved by using a variety of methods. The best results have been achieved with the combined excision of the diverticulum with cricopharyngeal myotomy. It has been our practice to do an open diverticu- lectomy over a bougie ranging from $40 \mathrm{~F}$ to $50 \mathrm{~F}$. This use of the bougie avoids excessive resection of the esophageal mucosa from traction on the diverticulum, which could lead to narrowing of the lumen. Modern-day staplers have been awkward to place flush with the bougie and have been avoided in favor of the open-hand 
sewn technique. The esophageal muscle has been closed over the suture line for a second layer of closure. The posterior edge of muscle can be difficult to identify. All techniques have a low failure rate. Nonetheless, recurrence can pose difficult challenges because of scarring, lack of tissue planes, and lack of available tissue for secondary coverage of the esophageal closure. In reoperation situations esophageal muscle might be unavailable. If esophageal muscle is used to cover the diverticulectomy site, performance of the myotomy and separation of the muscle from the mucosa can be awkward. The described technique avoids these problems.

We have found the currently described approach superior to the more traditional approach. The omohyoid muscle is readily available, and there is little noticeable functional loss if it is used as described. It provides an excellent second-layer buttress to the diverticulectomy suture line. By extending the myotomy from the base of the diverticulum, it allows for a more reliable myotomy and for better separation of the muscle from the mucosa. Inserting the omohyoid muscle between the cut edges of the cricopharyngeal muscle undergoing myotomy prevents these edges from coapting and causing restenosis.

The use of the omohyoid muscle is recommended as a second layer of closure in reoperations for Zenker diverticulum. Its use might also have advantages over the standard repairs for primary correction of Zenker diverticulum.

\section{References}

1. Ellis FH Jr. Pharyngoesophageal (Zenker's) diverticulum. Adv Surg. 1995;28:171-89.

2. Collard JM, Otte JB, Kestens PJ. Endoscopic stapling technique of esophagodiverticulostomy for Zenker's diverticulum. Ann Thorac Surg. 1993;56:573-6.

3. Duranceau A, Rheault MJ, Jamieson GG. Physiological response to cricopharyngeal myotomy and diverticulum suspension. Surgery. 1983;94:655-62.

4. Orringer MB. Extended cervical esophagomyotomy for cricopharyngeal dysfunction. J Thorac Cardiovasc Surg. 1980;80:669-78.

5. Payne SW, King RM. Pharyngoesophageal (Zenker's) diverticulum. Surg Clin North Am. 1983;63:815-24. 